\title{
Existence of solutions for Erdélyi-Kober fractional integral boundary value problems with $p(t)$-Laplacian operator
}

Xiaohui Shen ${ }^{1}$ and Tengfei Shen ${ }^{2^{*}}$

"Correspondence: stfcool@126.com

${ }^{2}$ School of Mathematics, China University of Mining and

Technology, Xuzhou 221116,

P.R. China

Full list of author information is

available at the end of the article

\section{Springer}

\begin{abstract}
This paper aims to consider the solvability for Erdélyi-Kober fractional integral boundary value problems with $p(t)$-Laplacian operator at resonance. By employing the coincidence degree method, some new results on the existence of solutions are acquired.
\end{abstract}

MSC: 34A08; 34B15

Keywords: Fractional differential equation; Boundary value problem; $p(t)$-Laplacian operator; Coincidence degree method

\section{Introduction}

In this paper, we consider the following fractional integral boundary value problem:

$$
\left\{\begin{array}{l}
D_{0+}^{\beta} \phi_{p(t)}\left(D_{0+}^{\alpha} x(t)\right)=f\left(t, x(t), D_{0+}^{\alpha-2} x(t), D_{0+}^{\alpha-1} x(t)\right), \quad t \in(0,1), \\
x(0)=0, \quad a_{1} D_{0+}^{\alpha-2} x(1)+b_{1} D_{0+}^{\alpha-1} x(1)=v_{1} I_{0+, \eta_{1}}^{\gamma_{1}, \delta_{1}} x\left(\xi_{1}\right), \\
D_{0+}^{\alpha} x(0)=0, \quad a_{2} D_{0+}^{\alpha-2} x(0)+b_{2} D_{0+}^{\alpha-1} x(0)=v_{2} I_{0+, \eta_{2}}^{\gamma_{2}, \delta_{2}} x\left(\xi_{2}\right),
\end{array}\right.
$$

where $D_{0+}^{\beta}$ and $D_{0+}^{\alpha}$ are Riemann-Liouville fractional derivatives with $0<\beta \leq 1$ and $2<$ $\alpha \leq 3, I_{0+, \eta_{i}}^{\gamma_{i}, \delta_{i}}$ is Erdélyi-Kober fractional integral of order $\delta_{i}>0$ with $\eta_{i}>0$ and $\gamma_{i}>0$ in which $i=1,2, f:[0,1] \times \mathbb{R}^{3} \rightarrow \mathbb{R}$ is continuous, $a_{i}, b_{i}, v_{i}$ are real numbers in which $i=1,2$, $0<\xi_{1}<\xi_{2}<1, \phi_{p(t)}(s)=|s|^{p(t)-2} s$ is $p(t)$-Laplacian operator with $p(t) \in C^{1}[0,1]$ and $p(t)>1$ for $s \in \mathbb{R}$. We always assume that the following condition holds:

$$
\begin{array}{ll}
(H) \quad & \left(a_{1}+b_{1}\right) \Gamma(\alpha) \Gamma\left(\gamma_{1}+\frac{\alpha-1}{\eta_{1}}+\delta_{1}+1\right)=v_{1} \xi_{1}^{\alpha-1} \Gamma\left(\gamma_{1}+\frac{\alpha-1}{\eta_{1}}+1\right) ; \\
& a_{1} \Gamma(\alpha-1) \Gamma\left(\gamma_{1}+\frac{\alpha-2}{\eta_{1}}+\delta_{1}+1\right)=v_{1} \xi_{1}^{\alpha-2} \Gamma\left(\gamma_{1}+\frac{\alpha-2}{\eta_{1}}+1\right) ; \\
& b_{2} \Gamma(\alpha) \Gamma\left(\gamma_{2}+\frac{\alpha-1}{\eta_{2}}+\delta_{2}+1\right)=v_{2} \xi_{2}^{\alpha-1} \Gamma\left(\gamma_{2}+\frac{\alpha-1}{\eta_{2}}+1\right) ; \\
& a_{2} \Gamma(\alpha-1) \Gamma\left(\gamma_{2}+\frac{\alpha-2}{\eta_{2}}+\delta_{2}+1\right)=v_{2} \xi_{2}^{\alpha-2} \Gamma\left(\gamma_{2}+\frac{\alpha-2}{\eta_{2}}+1\right) .
\end{array}
$$

(c) The Author(s) 2020. This article is licensed under a Creative Commons Attribution 4.0 International License, which permits use, sharing, adaptation, distribution and reproduction in any medium or format, as long as you give appropriate credit to the original author(s) and the source, provide a link to the Creative Commons licence, and indicate if changes were made. The images or other third party material in this article are included in the article's Creative Commons licence, unless indicated otherwise in a credit line to the material. If material is not included in the article's Creative Commons licence and your intended use is not permitted by statutory regulation or exceeds the permitted use, you will need to obtain permission directly from the copyright holder. To view a copy of this licence, visit http://creativecommons.org/licenses/by/4.0/. 
Fractional differential equations have a wide application background on many research fields such as physics, biology, electrical circuits, material, etc. (see [1-7]). For instance, Leszczynski and Blaszczyk [2] took advantage of a fractional differential model to show the height of granular material falling over time in a silo:

$$
{ }^{C} D_{T_{-}}^{\alpha} D_{a+}^{\alpha} h^{*}(t)+\beta h^{*}(t)=0, \quad t \in[0, T]
$$

where ${ }^{C} D_{T-}^{\alpha}$ represents left Caputo fractional derivative, $D_{a+}^{\alpha}$ means right Caputo fractional derivative with $\alpha \in(0,1), h^{*}(t)=h_{\text {bed }}-h(t)$ in which $h(t)$ acts as dropping height for silo emptying and $h_{\text {bed }}$ stands for the initial bed height. In recent years, more and more scholars have focused on investigating the existence and multiplicity of solutions to boundary value problems of fractional differential equations by various methods such as fixed point theory (see [8-10]), coincidence degree method (see [11-14]), critical point theory (see $[15,16])$, etc. For example, by the fixed point theorem for multivalued operators, Ahmad and Ntouyas [9] considered the existence of solutions to the following fractional differential inclusions with nonlocal multi-point Erdélyi-Kober fractional integral boundary value conditions:

$$
\left\{\begin{array}{l}
D_{0+}^{q} x(t) \in F(t, x(t))+G(t, x(t)), \quad t \in(0, T), \\
x(0)=0, \quad \alpha x(T)=\sum_{i=1}^{m} \beta_{i} I_{\eta_{i}}^{\gamma_{i}, \delta_{i}} x\left(\xi_{i}\right)
\end{array}\right.
$$

where $\alpha, \beta_{i} \in \mathbb{R}, \xi_{i} \in(0, T), i=1,2, \ldots, m, D_{0+}^{q}$ is Riemann-Liouville fractional derivative of order $q$ that $1<q \leq 2, I_{\eta_{i}}^{\gamma_{i}, \delta_{i}}$ is the Erdélyi-Kober fractional integral of order $\delta_{i}>0$ with $\eta_{i}>0$, and $\gamma_{i} \in \mathbb{R}, i=1,2, \ldots, m, F, G:[0, T] \times \mathbb{R} \rightarrow \mathcal{P}(\mathbb{R})$ are multivalued maps, where $\mathcal{P}(\mathbb{R})$ is the family of all nonempty subsets of $\mathbb{R}$.

As is known to all, the boundary value problem with $p$-Laplacian operator is a classical problem in differential equations of integer order (see [17-19] and the references therein). Recently, a growing number of scholars have devoted their attention to studying fractional boundary value problems with $p$-Laplacian operator (see [20-23]). For example, by constructing Green's functions and using some fixed point theorems, Mahmudov and Unul [20] considered the existence and uniqueness of solutions to integral boundary value problem of the following fractional differential equations with $p$-Laplacian operator:

$$
\left\{\begin{array}{l}
D_{0+}^{\beta} \phi_{p}\left(D_{0+}^{\alpha} x(t)\right)=f\left(t, x(t), D_{0+}^{\gamma} x(t)\right), \quad t \in[0,1] \\
x(0)+\mu_{1} x(1)=\sigma_{1} \int_{0}^{1} g(s, x(s)) d s \\
x^{\prime}(0)+\mu_{2} x^{\prime}(1)=\sigma_{2} \int_{0}^{1} h(s, x(s)) d s \\
D_{0+}^{\alpha} x(0)=0, \quad D_{0+}^{\alpha} x(1)=v D_{0+}^{\alpha} x(\eta)
\end{array}\right.
$$

where $D_{0_{+}}^{\alpha}, D_{0+}^{\beta}, D_{0+}^{\gamma}$ are Caputo fractional derivatives, $1<\alpha \leq 2,0<\beta, \gamma \leq 1,0<\eta<1$, $v, \mu_{i}, \sigma_{i}>0(i=1,2), \phi_{p}(\cdot)$ is a $p$-Laplacian operator, $f, g, h$ are continuous. After that, Shen and Liu [24] studied the following integral boundary value problem of fractional differential equations with $p(t)$-Laplacian operator at non-resonance or resonance:

$$
\left\{\begin{array}{l}
D_{0+}^{\beta} \phi_{p(t)}\left(D_{0+}^{\alpha} x(t)\right)+f(t, x(t))=0, \quad t \in(0,1), \\
x(0)=0, \quad D_{0+}^{\alpha-1} x(1)=\gamma I_{0+}^{\alpha-1} x(\eta), \quad D_{0+}^{\alpha} x(0)=0,
\end{array}\right.
$$


where $1<\alpha \leq 2,0<\beta \leq 1, D_{0+}^{\alpha}$ and $D_{0+}^{\beta}$ are Riemann-Liouville fractional derivatives, $\gamma>0,0<\eta<1, f:[0,1] \times \mathbb{R} \rightarrow \mathbb{R}$ is continuous. $\phi_{p(t)}(\cdot)$ is a $p(t)$-Laplacian operator with $p(t) \in C^{1}[0,1]$ and $p(t)>1$. Note that the dimension of the kernel of operator $D_{0+}^{\beta} \phi_{p(t)}\left(D_{0+}^{\alpha} x\right)$ is equal to 1 when $\gamma \eta^{2 \alpha-2}=\Gamma(2 \alpha-1)$ that is called the resonant case. By the coincidence degree method, the existence of solutions to problem (1.4) was obtained. It should be mentioned that the $p(t)$-Laplacian operator acts as the generalized operator which occurs in many research fields such as elasticity theory, image restoration, and nonlinear electrorheological fluids (see [25-27]). Moreover, for boundary value problems of differential equations of integer order with $p(t)$-Laplacian operator, please refer to [28, 29] and the references therein. Note that it is a nonstandard growth operator and can turn into the $p$-Laplacian operator when $p(t)=p$.

Motivated by the above work, in our paper we aim to study the existence of solutions to problem (1.1). It should be emphasized that the Erdélyi-Kober fractional integral operator is a generalization of the integral of integer order and can convert into Riemann-Liouville fractional integral with a power weight when $\eta=1$ and $\gamma=0$. So, a problem of this type becomes more interesting and challenging. Moreover, noting that the dimension of the kernel of operator $D_{0^{+}}^{\beta} \varphi_{p(t)}\left(D_{0^{+}}^{\alpha} x\right)$ is equal to 2 in (1.1), it will cause a lot of difficulties when we use the coincidence degree method such as constructing continuous linear projections. Thus, our results extend and enrich some existing papers. Furthermore, there are few papers studying fractional integral boundary value problem with $p(t)$-Laplacian operator.

\section{Preliminaries}

For the convenience of readers, some basic knowledge will be presented.

Definition 2.1 ([30]) Let $X$ and $Y$ be real Banach spaces, and let $L: \operatorname{dom} L \subset X \rightarrow Y$ be a linear operator. If $\operatorname{dim} \operatorname{Ker} L=\operatorname{codim} \operatorname{Im} L<+\infty$ and $\operatorname{Im} L$ is a closed subset in $Y$, then $L$ is a Fredholm operator with index zero. Define the continuous linear projections $P: X \rightarrow$ $X$ and $Q: Y \rightarrow Y$ that satisfy $\operatorname{Im} P=\operatorname{Ker} L$ and $\operatorname{Ker} Q=\operatorname{Im} L$; it follows that $\left.L\right|_{\operatorname{dom} L \cap \operatorname{Ker} P}$ : $\operatorname{dom} L \cap \operatorname{Ker} P \rightarrow \operatorname{Im} L$ is reversible. Denote its inverse map by $K_{P}$, and let $K_{P, Q}=K_{P}(I-Q)$. If $\Omega$ is an open bounded subset of $X$ and $\operatorname{dom} L \cap \Omega \neq \varnothing$, the map $N$ is $L$-compact on $\bar{\Omega}$ when $Q N: \bar{\Omega} \rightarrow Y$ is bounded and $K_{P}(I-Q) N: \bar{\Omega} \rightarrow X$ is compact.

Lemma 2.2 ([30]) Let $L: \operatorname{dom} L \subset X \rightarrow Y$ be a Fredholm operator of index zero and $N$ : $X \rightarrow Y$ be L-compact on $\bar{\Omega}$. Assume that the following conditions are satisfied:

(i) $L x \neq \lambda N x$ for every $(x, \lambda) \in[(\operatorname{dom} L \backslash \operatorname{Ker} L)] \cap \partial \Omega \times(0,1)$;

(ii) $N x \notin \operatorname{Im} L$ for every $x \in \operatorname{Ker} L \cap \partial \Omega$;

(iii) $\operatorname{deg}\left(\left.Q N\right|_{\operatorname{Ker} L}, \operatorname{Ker} L \cap \Omega, 0\right) \neq 0$, where $Q: Y \rightarrow Y$ is a projection such that $\operatorname{Im} L=\operatorname{Ker} Q$.

Then the equation $L x=N x$ has at least one solution in $\operatorname{dom} L \cap \bar{\Omega}$.

Definition 2.3 ([1]) The Riemann-Liouville fractional integral of order $\alpha>0$ for the function $x:(0,+\infty) \rightarrow \mathbb{R}$ is defined by

$$
I_{0+}^{\alpha} x(t)=\frac{1}{\Gamma(\alpha)} \int_{0}^{t}(t-s)^{\alpha-1} x(s) \mathrm{d} s
$$


provided the right-hand side is pointwise defined on $(0,+\infty)$, where $\Gamma(\alpha)$ is the standard gamma function.

Definition 2.4 ([1]) The Riemann-Liouville fractional derivative of order $\alpha>0$ of $x$ : $(0,+\infty) \rightarrow \mathbb{R}$ is defined by

$$
D_{0+}^{\alpha} u(t)=\frac{1}{\Gamma(n-\alpha)}\left(\frac{d}{d t}\right)^{n} \int_{0}^{t}(t-s)^{n-\alpha-1} x(s) d s,
$$

provided the right-hand side integral is pointwise defined on $(0,+\infty)$, where $n=[\alpha]+1$.

Definition 2.5 ([1]) The Erdlyi-Kober fractional integral of order $\delta>0$ with $\eta>0$ and $\gamma \in \mathbb{R}$ of a continuous function $x:(0, \infty) \rightarrow \mathbb{R}$ is defined by

$$
I_{0+, \eta}^{\gamma, \delta} x(t)=\frac{\eta t^{-\eta(\delta+\gamma)}}{\Gamma(\delta)} \int_{0}^{t} \frac{s^{\eta \gamma+\eta-1} x(s)}{\left(t^{\eta}-s^{\eta}\right)^{1-\delta}} d s,
$$

provided the right-hand side is pointwise defined on $(0,+\infty)$.

Remark 2.6 If $\eta=1, \gamma>0$, and $\delta>0$, the above operator changes into the Kober operator (see [31]). Moreover, if $\gamma=0$, the Kober operator turns into the following RiemannLiouville fractional integral with a power weight:

$$
I_{0+, 1}^{1, \delta} x(t)=\frac{t^{-\delta}}{\Gamma(\delta)} \int_{0}^{t}(t-s)^{\delta-1} x(s) d s, \quad \delta>0 .
$$

Lemma 2.7 ([1]) If $x(t) \in L_{p}(0,1)(1 \leq p \leq \infty)$, then

(i) $D_{0+}^{\alpha} I_{0+}^{\alpha} x(t)=x(t)$ with $\alpha>0$ holds almost everywhere on $[0,1]$;

(ii) $D_{0+}^{\beta} I_{0+}^{\alpha} x(t)=I_{0+}^{\alpha-\beta} x(t)$ with $\alpha>\beta>0$ holds almost everywhere on $[0,1]$.

Lemma 2.8 ([1]) Let $\alpha \geq 0, m \in \mathbb{N}$, and $D=d / d t$. If the fractional derivatives $D_{0+}^{\alpha} x(t)$ and $D_{0+}^{\alpha+m} x(t)$ exist, then

$$
D^{m} D_{0+}^{\alpha} x(t)=D_{0+}^{\alpha+m} x(t) .
$$

Lemma 2.9 ([1]) The following equalities hold for fractional integral and derivative:

(i) If $\alpha \geq 0, \lambda>-1, \lambda \neq \alpha-i, i=1,2, \ldots,[\alpha]+1$, we have

$$
D_{0+}^{\alpha} t^{\lambda}=\frac{\Gamma(\lambda+1)}{\Gamma(\lambda-\alpha+1)} t^{\lambda-\alpha} .
$$

$$
\text { Moreover, } D_{0+}^{\alpha} t^{\alpha-i}=0, i=1,2, \ldots,[\alpha]+1 \text {. }
$$

(ii) If $\alpha>0, \lambda>-1$, we have

$$
I_{0+}^{\alpha} t^{\lambda}=\frac{\Gamma(\lambda+1)}{\Gamma(\lambda+\alpha+1)} t^{\lambda+\alpha} .
$$

(iii) If $\delta, \eta, \alpha>0, \gamma \geq 0$, we have

$$
I_{0+, \eta}^{\gamma, \delta} t^{\alpha}=\frac{t^{\alpha} \Gamma(\gamma+(\alpha / \eta)+1)}{\Gamma(\gamma+(\alpha / \eta)+\delta+1)} .
$$


Lemma 2.10 ([1]) Assume that $x \in C(0,1) \cap L^{1}(0,1)$ with a fractional derivative of order $\alpha>0$ which belongs to $C(0,1) \cap L^{1}(0,1)$. Then

$$
I_{0+}^{\alpha} D_{0+}^{\alpha} x(t)=x(t)+c_{1} t^{\alpha-1}+c_{2} t^{\alpha-2}+\cdots+c_{\mathbb{N}} t^{\alpha-\mathbb{N}}
$$

for some $c_{i} \in \mathbb{R}, i=1,2, \ldots, \mathbb{N}$, where $\mathbb{N}=[\alpha]+1$.

Lemma 2.11 ([29]) For any $(t, x) \in[0,1] \times \mathbb{R}, \phi_{p(t)}(x)=|x|^{p(t)-2} x$ is a homeomorphism from $\mathbb{R}$ to $\mathbb{R}$. Moreover, it is strictly monotone increasing for any fixed $t$. Furthermore, for any $t \in[0,1]$, its inverse operator $\phi_{p(t)}^{-1}(\cdot)$ is defined by

$$
\begin{cases}\phi_{p(t)}^{-1}(x)=|x|^{\frac{2-p(t)}{p(t)-1}} x, & x \in \mathbb{R} \backslash\{0\} \\ \phi_{p(t)}^{-1}(0)=0, & x=0,\end{cases}
$$

that is continuous and sends bounded sets into bounded sets.

In order to make the continuation theorem of Mawhin applicable, the following lemma needs to be established.

Lemma 2.12 Problem (1.1) is equivalent to the following fractional integral boundary value problem:

$$
\left\{\begin{array}{l}
D_{0+}^{\alpha} x(t)=\phi_{p(t)}^{-1}\left(I_{0+}^{\beta} f\left(t, x(t), D_{0+}^{\alpha-2} x(t), D_{0+}^{\alpha-1} x(t)\right)\right), \quad t \in(0,1), \\
x(0)=0, \quad a_{1} D_{0+}^{\alpha-2} x(1)+b_{1} D_{0+}^{\alpha-1} x(1)=v_{1} I_{0+, \eta_{1}}^{\gamma_{1}, \delta_{1}} x\left(\xi_{1}\right), \\
a_{2} D_{0+}^{\alpha-2} x(0)+b_{2} D_{0+}^{\alpha-1} x(0)=v_{2} I_{0+, \eta_{2}}^{\gamma_{2}, \delta_{2}} x\left(\xi_{2}\right) .
\end{array}\right.
$$

Proof Firstly, since $D_{0+}^{\alpha} x(0)=0$, it is clear that problem (1.1) implies (2.1). On the other hand, taking $t=0$ into the following equality

$$
D_{0+}^{\alpha} x(t)=\phi_{p(t)}^{-1}\left(I_{0+}^{\beta} f\left(t, x(t), D_{0+}^{\alpha-2} x(t), D_{0+}^{\alpha-1} x(t)\right)\right),
$$

we have $D_{0+}^{\alpha} x(0)=0$. Additionally, making the operators $\phi_{p(t)}$ and $D_{0+}^{\beta}$ act on both sides of the above equality, it follows that $D_{0+}^{\beta} \phi_{p(t)}\left(D_{0+}^{\alpha} x(t)\right)=f\left(t, x(t), D_{0+}^{\alpha-2} x(t), D_{0+}^{\alpha-1} x(t)\right)$. Thus, problem (2.1) implies (1.1).

\section{Main result}

Let $Y=C[0,1]$ with the norm $\|y\|_{\infty}=\max _{t \in[0,1]}|y(t)|, X=\left\{x \mid x, D_{0+}^{\alpha-2} x, D_{0+}^{\alpha-1} x \in C[0,1]\right\}$ with the norm $\|x\|_{X}=\max \left\{\|x\|_{\infty},\left\|D_{0+}^{\alpha-2} x\right\|_{\infty},\left\|D_{0+}^{\alpha-1} x\right\|_{\infty}\right\}$. Clearly, $X$ and $Y$ are Banach spaces. Based on Lemma 2.12, we just need to consider the existence of solutions to problem (2.1). Define the operator $L: \operatorname{dom} L \subset X \rightarrow Y$ by

$$
L x=D_{0+}^{\alpha} x(t),
$$

where

$$
\begin{aligned}
\operatorname{dom} L= & \left\{x \in X \mid D_{0+}^{\alpha} x(t) \in Y, x(0)=0, a_{1} D_{0+}^{\alpha-2} x(1)+b_{1} D_{0+}^{\alpha-1} x(1)=v_{1} I_{0+, \eta_{1}}^{\gamma_{1}, \delta_{1}} x\left(\xi_{1}\right)\right. \\
& \left.a_{2} D_{0+}^{\alpha-2} x(0)+b_{2} D_{0+}^{\alpha-1} x(0)=v_{2} I_{0+, \eta_{2}}^{\gamma_{2}, \delta_{2}} x\left(\xi_{2}\right)\right\} .
\end{aligned}
$$


Moreover, define $N: X \rightarrow Y$ by

$$
N x(t)=\phi_{p(t)}^{-1}\left(I_{0+}^{\beta} f\left(t, x(t), D_{0+}^{\alpha-2} x(t), D_{0+}^{\alpha-1} x(t)\right)\right), \quad \forall t \in[0,1] .
$$

Then problem (1.1) is equivalent to the following operator equation:

$$
L x=N x, \quad x \in \operatorname{dom} L .
$$

Define the operators $\Phi_{1}, \Phi_{2}: Y \rightarrow Y$ by

$$
\begin{aligned}
& \Phi_{1} y=b_{1} I_{0+}^{1} y(1)+a_{1} I_{0+}^{2} y(1)-v_{1} I_{0+, \eta_{1}}^{\gamma_{1}, \delta_{1}} I_{0+}^{\alpha} y\left(\xi_{1}\right), \\
& \Phi_{2} y=I_{0+, \eta_{2}}^{\gamma_{2}, \delta_{2}} I_{0+}^{\alpha} y\left(\xi_{2}\right) .
\end{aligned}
$$

Next, some important lemmas will be presented before establishing main conclusions.

Lemma 3.1 Let L be given by (3.1), then

$$
\begin{aligned}
& \operatorname{Ker} L=\left\{x \in X \mid x(t)=c_{1} t^{\alpha-1}+c_{2} t^{\alpha-2}, c_{1}, c_{2} \in \mathbb{R}, \forall t \in[0,1]\right\} \\
& \operatorname{Im} L=\left\{y \in Y \mid \Phi_{1} y=\Phi_{2} y=0\right\} .
\end{aligned}
$$

Proof It is clear that (3.2) is satisfied, which is linearly homeomorphic to $\mathbb{R}^{2}$. If $y \in \operatorname{Im} L$, we can find a function $x \in \operatorname{dom} L$ such that $y(t)=D_{0^{+}}^{\alpha} x(t)$. Based on Lemma 2.2 and Lemma 2.9, it follows

$$
\begin{aligned}
& x(t)=I_{0^{+}}^{\alpha} y(s)+c_{1} t^{\alpha-1}+c_{1} t^{\alpha-2}, \\
& D_{0^{+}}^{\alpha-2} x(t)=I_{0^{+}}^{2} y(s)+c_{1} \Gamma(\alpha) t+c_{2} \Gamma(\alpha-1), \\
& D_{0^{+}}^{\alpha-1} x(t)=I_{0^{+}}^{1} y(s)+c_{1} \Gamma(\alpha),
\end{aligned}
$$

which together with the boundary conditions

$$
\begin{aligned}
& a_{1} D_{0+}^{\alpha-2} x(1)+b_{1} D_{0+}^{\alpha-1} x(1)=v_{1} I_{0+, \eta_{1}}^{\gamma_{1}, \delta_{1}} x\left(\xi_{1}\right), \\
& a_{2} D_{0+}^{\alpha-2} x(0)+b_{2} D_{0+}^{\alpha-1} x(0)=v_{2} I_{0+, \eta_{2}}^{\gamma_{2}, \delta_{2}} x\left(\xi_{2}\right)
\end{aligned}
$$

yields that

$$
\begin{aligned}
& b_{1} I_{0+}^{1} y(1)+a_{1} I_{0+}^{2} y(1)-v_{1} I_{0+, y_{1}}^{\gamma_{1}, \delta_{1}} I_{0+}^{\alpha} y\left(\xi_{1}\right)+\left(\left(a_{1}+b_{1}\right) \Gamma(\alpha)-\frac{v_{1} \xi_{1}^{\alpha-1} \Gamma\left(\gamma_{1}+\frac{\alpha-1}{\eta_{1}}+1\right)}{\Gamma\left(\gamma_{1}+\frac{\alpha-1}{\eta_{1}}+\delta_{1}+1\right)}\right) c_{1} \\
& \quad+\left(a_{1} \Gamma(\alpha-1)-\frac{\nu_{1} \xi_{1}^{\alpha-2} \Gamma\left(\gamma_{1}+\frac{\alpha-2}{\eta_{1}}+1\right)}{\Gamma\left(\gamma_{1}+\frac{\alpha-2}{\eta_{1}}+\delta_{1}+1\right)}\right) c_{2}=0
\end{aligned}
$$

and

$$
\begin{gathered}
I_{0+, y_{2}}^{\gamma_{2}, \delta_{2}} I_{0+}^{\alpha} y\left(\xi_{2}\right)+\left(b_{2} \Gamma(\alpha)-\frac{\nu_{2} \xi_{2}^{\alpha-1} \Gamma\left(\gamma_{2}+\frac{\alpha-1}{\eta_{2}}+1\right)}{\Gamma\left(\gamma_{2}+\frac{\alpha-1}{\eta_{2}}+\delta_{2}+1\right)}\right) c_{1} \\
+\left(a_{2} \Gamma(\alpha-1)-\frac{\nu_{2} \xi_{2}^{\alpha-2} \Gamma\left(\gamma_{2}+\frac{\alpha-2}{\eta_{2}}+1\right)}{\Gamma\left(\gamma_{2}+\frac{\alpha-2}{\eta_{2}}+\delta_{2}+1\right)}\right) c_{2}=0 .
\end{gathered}
$$


By $(H)$, one has $\Phi_{j} y(t)=0, j=1,2$.

On the other hand, if $y$ belongs to $Y$ and satisfies $\Phi_{j} y(t)=0, j=1,2$, setting $x(t)=I_{0^{+}}^{\alpha} y(t)$, one has $x \in \operatorname{dom} L$ and $L x(t)=D_{0^{+}}^{\alpha} x(t)=y(t)$. Thus, $y \in \operatorname{Im} L$ and (3.3) holds.

For convenience, the following notations are given:

$$
\begin{aligned}
& \Delta_{1}:=\frac{b_{1}}{\alpha}+\frac{a_{1}}{\alpha(1+\alpha)}-\frac{\nu_{1} \xi_{1}^{\alpha+\beta-1} \Gamma(\alpha) \Gamma\left(\gamma_{1}+1+\frac{\alpha+\beta-1}{\eta_{1}}\right)}{\Gamma(\alpha+\beta) \Gamma\left(\gamma_{1}+\delta_{1}+1+\frac{\alpha+\beta-1}{\eta_{1}}\right)} \\
& \Delta_{2}:=\frac{\xi_{2}^{\alpha+\beta-1} \Gamma(\alpha) \Gamma\left(\gamma_{2}+1+\frac{\alpha+\beta-1}{\eta_{2}}\right)}{\Gamma(\alpha+\beta) \Gamma\left(\gamma_{2}+\delta_{2}+1+\frac{\alpha+\beta-1}{\eta_{2}}\right)}, \\
& \Delta_{3}:=\frac{b_{1}}{\alpha-1}+\frac{a_{1}}{\alpha(\alpha-1)}-\frac{\nu_{1} \xi_{1}^{\alpha+\beta-2} \Gamma(\alpha-1) \Gamma\left(\gamma_{1}+1+\frac{\alpha+\beta-2}{\eta_{1}}\right)}{\Gamma(\alpha+\beta-1) \Gamma\left(\gamma_{1}+\delta_{1}+1+\frac{\alpha+\beta-2}{\eta_{1}}\right)} \\
& \Delta_{4}:=\frac{\xi_{2}^{\alpha+\beta-2} \Gamma(\alpha-1) \Gamma\left(\gamma_{2}+1+\frac{\alpha+\beta-2}{\eta_{2}}\right)}{\Gamma(\alpha+\beta-1) \Gamma\left(\gamma_{2}+\delta_{2}+1+\frac{\alpha+\beta-2}{\eta_{2}}\right)} \\
& \Delta_{(}:=\left|\begin{array}{ll}
\Delta_{1} & \Delta_{2} \\
\Delta_{3} & \Delta_{4}
\end{array}\right| .
\end{aligned}
$$

Lemma 3.2 If $\Delta \neq 0$, the continuous linear projection operators $P: X \rightarrow X$ and $Q: Y \rightarrow Y$ can be written as

$$
\begin{aligned}
& P x(t)=\frac{1}{\Gamma(\alpha)} D_{0+}^{\alpha-1} x(0) t^{\alpha-1}+\frac{1}{\Gamma(\alpha-1)} D_{0+}^{\alpha-2} x(0) t^{\alpha-2}, \quad t \in[0,1], \\
& Q y(t)=\left(\Upsilon_{1} y(t)\right) t^{\alpha-1}+\left(\Upsilon_{2} y(t)\right) t^{\alpha-2}, \quad t \in[0,1]
\end{aligned}
$$

where

$$
\Upsilon_{1} y(t)=\frac{1}{\Delta}\left(\Delta_{4} \Phi_{1} y(t)-\Delta_{3} \Phi_{2} y(t)\right), \quad \Upsilon_{2} y(t)=\frac{1}{\Delta}\left(-\Delta_{2} \Phi_{1} y(t)+\Delta_{1} \Phi_{2} y(t)\right) .
$$

Moreover, $L$ is a Fredholm operator of index zero and $K_{P}: \operatorname{Im} L \rightarrow \operatorname{dom} L \cap \operatorname{Ker} P$ can be presented as follows:

$$
K_{P} y(t)=\frac{1}{\Gamma(\alpha)} \int_{0}^{t}(t-s)^{\alpha-1} y(s) \mathrm{d} s, \quad \forall t \in[0,1]
$$

Proof For the operator $P$, it is clear that for $x \in X, P^{2} x=P x, \operatorname{Im} P=\operatorname{Ker} L$, and $X=\operatorname{Ker} L \oplus$ $\operatorname{Ker} P$. For the operator $Q$, if $y \in Y$, then

$$
\begin{aligned}
\Upsilon_{1}\left(\left(\Upsilon_{1} y(t)\right) t^{\alpha-1}\right) & =\frac{1}{\Delta}\left(\Delta_{4} \Phi_{1}\left(\left(\Upsilon_{1} y(t)\right) t^{\alpha-1}\right)-\Delta_{3} \Phi_{2}\left(\left(\Upsilon_{1} y(t)\right) t^{\alpha-1}\right)\right) \\
& =\frac{\Upsilon_{1} y}{\Delta}\left(\Delta_{4} \Phi_{1}\left(t^{\alpha-1}\right)-\Delta_{3} \Phi_{2}\left(t^{\alpha-1}\right)\right) \\
& =\frac{1}{\Delta}\left(\Delta_{4} \Delta_{1}-\Delta_{3} \Delta_{2}\right) \Upsilon_{1} y=\Upsilon_{1} y,
\end{aligned}
$$




$$
\begin{aligned}
\Upsilon_{1}\left(\left(\Upsilon_{2} y(t)\right) t^{\alpha-2}\right) & =\frac{1}{\Delta}\left(\Delta_{4} \Phi_{1}\left(\left(\Upsilon_{2} y(t)\right) t^{\alpha-2}\right)-\Delta_{3} \Phi_{2}\left(\left(\Upsilon_{2} y(t)\right) t^{\alpha-2}\right)\right) \\
& =\frac{\Upsilon_{2} y}{\Delta}\left(\Delta_{4} \Phi_{1}\left(t^{\alpha-2}\right)-\Delta_{3} \Phi_{2}\left(t^{\alpha-2}\right)\right) \\
& =\frac{1}{\Delta}\left(\Delta_{4} \Delta_{3}-\Delta_{3} \Delta_{4}\right) \Upsilon_{2} y=0, \\
\Upsilon_{2}\left(\left(\Upsilon_{1} y(t)\right) t^{\alpha-1}\right) & =\frac{1}{\Delta}\left(-\Delta_{2} \Phi_{1}\left(\left(\Upsilon_{1} y(t)\right) t^{\alpha-1}\right)+\Delta_{1} \Phi_{2}\left(\left(\Upsilon_{1} y(t)\right) t^{\alpha-1}\right)\right) \\
& =\frac{\Upsilon_{1} y}{\Delta}\left(-\Delta_{2} \Phi_{1}\left(t^{\alpha-1}\right)+\Delta_{1} \Phi_{2}\left(t^{\alpha-1}\right)\right) \\
& =\frac{1}{\Delta}\left(-\Delta_{2} \Delta_{1}+\Delta_{1} \Delta_{2}\right) \Upsilon_{1} y=0, \\
\Upsilon_{2}\left(\left(\Upsilon_{2} y(t)\right) t^{\alpha-2}\right) & =\frac{1}{\Delta}\left(-\Delta_{2} \Phi_{1}\left(\left(\Upsilon_{2} y(t)\right) t^{\alpha-2}\right)+\Delta_{1} \Phi_{2}\left(\left(\Upsilon_{2} y(t)\right) t^{\alpha-2}\right)\right) \\
& =\frac{\Upsilon_{2} y}{\Delta}\left(-\Delta_{2} \Phi_{1}\left(t^{\alpha-2}\right)+\Delta_{1} \Phi_{2}\left(t^{\alpha-2}\right)\right) \\
& =\frac{1}{\Delta}\left(-\Delta_{2} \Delta_{3}+\Delta_{1} \Delta_{4}\right) \Upsilon_{2} y=\Upsilon_{2} y .
\end{aligned}
$$

Thus, $Q^{2} y=Q y$. Next, we will show $\operatorname{Ker} Q=\operatorname{Im} L$. In fact, if $y \in \operatorname{Ker} Q \subset Y$, we can get $\Upsilon_{1} y=\Upsilon_{2} y=0$, i.e.,

$$
\Delta_{4} \Phi_{1} y(t)-\Delta_{3} \Phi_{2} y(t)=0, \quad-\Delta_{2} \Phi_{1} y(t)+\Delta_{1} \Phi_{2} y(t)=0
$$

which together with $\Delta \neq 0$ yields $\Phi_{1} y(t)=\Phi_{2} y(t)=0$ and $\operatorname{Ker} Q \subset \operatorname{Im} L . \operatorname{If} y \in \operatorname{Im} L \subset Y$, from (3.2), it is clear that $\operatorname{Im} L \subset \operatorname{Ker} Q$. Thus, $\operatorname{Im} L=\operatorname{Ker} Q$, which together with $Q^{2} y=Q y$ implies $Y=\operatorname{Im} L \oplus \operatorname{Im} Q$. Thus, the operators $P$ and $Q$ are well defined. Moreover,

$$
\operatorname{dim} \operatorname{Ker} L=\operatorname{dim} \operatorname{Im} Q=\operatorname{codim} \operatorname{Im} L=2,
$$

which means that $L$ is a Fredholm operator of index zero.

Finally, $K_{P} x=\left(\left.L\right|_{\operatorname{dom} L \cap \operatorname{Ker} P}\right)^{-1}(x)$ will be proved. On the one hand, if $x \in \operatorname{dom} L \cap \operatorname{Ker} P$, one has

$$
D_{0^{+}}^{\alpha-1} x(0)=D_{0^{+}}^{\alpha-2} x(0)=0,
$$

which together with $x(0)=0$ yields $K_{P} L x(t)=I_{0+}^{\alpha} D_{0+}^{\alpha} x(t)=x(t)$. On the other hand, if $y \in$ $\operatorname{Im} L$, it is clear that $L K_{P} y=D_{0_{+}}^{\alpha} I_{0+}^{\alpha} y=y$. The proof is complete.

Theorem 3.3 Assume $\Delta \neq 0$ and the following conditions hold:

(H1) There exist nonnegative functions $h_{i} \in C[0,1], i=1,2,3,4$, such that, for any $t \in$ $[0,1],(x, y, z) \in \mathbb{R}^{3}$,

$$
|f(t, x, y, z)| \leq h_{1}(t)+h_{2}(t)|x|^{\theta-1}+h_{3}(t)|y|^{\theta-1}+h_{4}(t)|z|^{\theta-1}, \quad 1<\theta \leq P_{m},
$$

where $P_{m}=\min _{t \in[0,1]} p(t)$. 
(H2) For $x \in \operatorname{dom} L$, there exists a constant $B_{1}>0$ such that if $\left|D_{0+}^{\alpha-2} x(t)\right|>B_{1}$ for any $t \in\left[\xi_{2}, 1\right]$, either

$$
\operatorname{sgn}\left\{D_{0+}^{\alpha-2} x(t)\right\} \Phi_{2} N x(t)>0 \quad \text { or } \quad \operatorname{sgn}\left\{D_{0+}^{\alpha-2} x(t)\right\} \Phi_{2} N x(t)<0 .
$$

(H3) For $x \in \operatorname{dom} L$, there exists a constant $B_{2}>0$ such that if $\left|D_{0+}^{\alpha-1} x(t)\right|>B_{2}$ for any $t \in\left[0, \xi_{1}\right]$, either

$$
\operatorname{sgn}\left\{D_{0+}^{\alpha-1} x(t)\right\} \Phi_{1} N x(t)>0 \quad \text { or } \quad \operatorname{sgn}\left\{D_{0+}^{\alpha-1} x(t)\right\} \Phi_{1} N x(t)<0 .
$$

Then problem (1.1) admits at least one solution, provided that

$$
\frac{2^{\theta}}{\Gamma(\beta+1)}\left(\frac{\left\|h_{2}\right\|_{\infty}}{(\Gamma(\alpha-1))^{\theta-1}}+\left\|h_{3}\right\|_{\infty}+\left\|h_{4}\right\|_{\infty}\right)<1
$$

Proof Let

$$
\begin{aligned}
& \Omega_{1}=\{x \in \operatorname{dom} L \backslash \operatorname{Ker} L \mid L x=\lambda N x, \lambda \in(0,1)\}, \\
& \Omega_{2}=\{x \mid x \in \operatorname{Ker} L, N x \in \operatorname{Im} L\}, \\
& \Omega_{3}=\left\{x \in \operatorname{Ker} L \mid \lambda J^{-1} x+(1-\lambda) Q N x=0, \lambda \in[0,1]\right\} \text { and } \\
& \Omega_{3}^{\prime}=\left\{x \in \operatorname{Ker} L \mid-\lambda J^{-1} x+(1-\lambda) Q N x=0, \lambda \in[0,1]\right\},
\end{aligned}
$$

where $J^{-1}: \operatorname{Ker} L \rightarrow \operatorname{Im} Q$ is defined by $J\left(c_{1} t^{\alpha-1}+c_{2} t^{\alpha-2}\right)=\frac{1}{\Delta}\left(\Delta_{4} c_{1}-\Delta_{3} c_{2}\right) t^{\alpha-1}+$ $\frac{1}{\Delta}\left(-\Delta_{2} c_{1}+\Delta_{1} c_{2}\right) t^{\alpha-2}, c_{1}, c_{2} \in \mathbb{R}$.

For any $x \in \Omega_{1}$, clearly, $N x \in \operatorname{Im} L=\operatorname{Ker} Q$ and $Q N x=0$, which implies $\Phi_{1} N x=\Phi_{2} N x=0$. In view of $(H 2)$ and $(H 3)$, we can find two constants $\mu_{1}, \mu_{2} \in[0,1]$ such that $\left|D_{0^{+}}^{\alpha-2} x\left(\mu_{1}\right)\right| \leq$ $B_{1},\left|D_{0^{+}}^{\alpha-1} x\left(\mu_{2}\right)\right| \leq B_{2}$. Thus, from Lemma 2.8 , one has

$$
\begin{aligned}
& D_{0+}^{\alpha-2} x(t)=D_{0+}^{\alpha-2} x\left(\mu_{1}\right)+\int_{\mu_{1}}^{t} D_{0+}^{\alpha-1} x(t) d t, \\
& D_{0+}^{\alpha-1} x(t)=D_{0+}^{\alpha-1} x\left(\mu_{2}\right)+\int_{\mu_{2}}^{t} D_{0+}^{\alpha} x(t) d t,
\end{aligned}
$$

which leads to $\left\|D_{0_{+}}^{\alpha-1} x\right\|_{\infty} \leq B_{2}+\left\|D_{0_{+}}^{\alpha} x\right\|_{\infty}$ and $\left\|D_{0_{+}}^{\alpha-2} x\right\|_{\infty} \leq B_{2}+B_{1}+\left\|D_{0_{+}}^{\alpha} x\right\|_{\infty}$. Moreover, based on $x(0)=0$, it follows that

$$
x(t)=I_{0+}^{\alpha-2} D_{0+}^{\alpha-2} x(t)
$$

which yields

$$
|x(t)| \leq \frac{1}{\Gamma(\alpha-2)} \int_{0}^{t}(t-s)^{\alpha-3} d t\left\|D_{0+}^{\alpha-2} x\right\|_{\infty} \leq \frac{1}{\Gamma(\alpha-1)}\left\|D_{0+}^{\alpha-2} x\right\|_{\infty} .
$$

Thus, $\|x\|_{\infty} \leq \frac{B_{1}+B_{2}}{\Gamma(\alpha-1)}+\frac{\left\|D_{0+}^{\alpha} x\right\|_{\infty}}{\Gamma(\alpha-1)}$. From $L u=\lambda N u$, we know that

$$
D_{0+}^{\alpha} x(t)=\lambda \phi_{p(t)}^{-1}\left(I_{0+}^{\beta} f\left(t, x(t), D_{0+}^{\alpha-2} x(t), D_{0+}^{\alpha-1} x(t)\right)\right) .
$$


Taking the operator $\phi_{p(t)}$ act on both sides of the above equality, we have

$$
\phi_{p(t)}\left(D_{0+}^{\alpha} x(t)\right)=\lambda^{p(t)-1}\left(I_{0+}^{\beta} f\left(t, x(t), D_{0+}^{\alpha-2} x(t), D_{0+}^{\alpha-1} x(t)\right)\right) .
$$

In view of $(H 1)$ and $\lambda \in(0,1)$, one has

$$
\begin{aligned}
\left|D_{0+}^{\alpha} x(t)\right|^{p(t)-1} \leq & \frac{1}{\Gamma(\beta)} \int_{0}^{t}(t-s)^{\beta-1}\left|f\left(t, x(t), D_{0+}^{\alpha-2} x(t), D_{0+}^{\alpha-1} x(t)\right)\right| d s \\
\leq & \frac{1}{\Gamma(\beta+1)}\left(\left\|h_{1}\right\|_{\infty}+\left\|h_{2}\right\|_{\infty}\|x\|_{\infty}^{\theta-1}+\left\|h_{3}\right\|_{\infty}\left\|D_{0+}^{\alpha-2} x\right\|_{\infty}^{\theta-1}\right. \\
& \left.+\left\|h_{4}\right\|_{\infty}\left\|D_{0+}^{\alpha-1} x\right\|_{\infty}^{\theta-1}\right) \\
\leq & \frac{1}{\Gamma(\beta+1)}\left(\left\|h_{1}\right\|_{\infty}+\left\|h_{2}\right\|_{\infty}\left(\frac{B_{1}+B_{2}}{\Gamma(\alpha-1)}+\frac{1}{\Gamma(\alpha-1)}\left\|D_{0+}^{\alpha} u\right\|_{\infty}\right)^{\theta-1}\right. \\
& \left.+\left\|h_{3}\right\|_{\infty}\left(B_{1}+B_{2}+\left\|D_{0+}^{\alpha} u\right\|_{\infty}\right)^{\theta-1}+\left\|h_{4}\right\|_{\infty}\left(B_{2}+\left\|D_{0+}^{\alpha} u\right\|_{\infty}\right)^{\theta-1}\right),
\end{aligned}
$$

which together with the basic inequality $(x+y)^{p} \leq 2^{p}\left(x^{p}+y^{p}\right), x, y, p>0$ yields

$$
\left|D_{0+}^{\alpha} x(t)\right|^{p(t)-1} \leq K_{1}+K_{2}\left\|D_{0+}^{\alpha} x\right\|_{\infty}^{\theta-1},
$$

where

$$
\begin{aligned}
& K_{1}=\frac{2^{\theta-1}}{\Gamma(\beta+1)}\left(\left\|h_{1}\right\|_{\infty}+\left(\frac{B_{1}+B_{2}}{\Gamma(\alpha-1)}\right)^{\theta-1}\left\|h_{2}\right\|_{\infty}+\left(B_{1}+B_{2}\right)^{\theta-1}\left\|h_{3}\right\|_{\infty}+B_{2}^{\theta-1}\left\|h_{4}\right\|_{\infty}\right), \\
& K_{2}=\frac{2^{\theta-1}}{\Gamma(\beta+1)}\left(\frac{1}{(\Gamma(\alpha-1))^{\theta-1}}\left\|h_{2}\right\|_{\infty}+\left\|h_{3}\right\|_{\infty}+\left\|h_{4}\right\|_{\infty}\right) .
\end{aligned}
$$

Thus, it follows that

$$
\left\|D_{0+}^{\alpha} x\right\|_{\infty} \leq 2^{\frac{1}{p(t)-1}}\left(K_{1}^{\frac{1}{p(t)-1}}+K_{2}^{\frac{1}{p(t)-1}}\left\|D_{0+}^{\alpha} x\right\|_{\infty}^{\frac{\theta-1}{p(t)-1}}\right) .
$$

Clearly, $\frac{\theta-1}{p(t)-1} \in(0,1]$, based on the basic inequality $x^{l} \leq x+1$, for $x>0, l \in(0,1]$, we can obtain that

$$
\left\|D_{0+}^{\alpha} x\right\|_{\infty} \leq 2^{\frac{1}{p(t)-1}} K_{1}^{\frac{1}{p(t)-1}}+2^{\frac{1}{p(t)-1}} K_{2}^{\frac{1}{p(t)-1}}\left(\left\|D_{0_{+}}^{\alpha} x\right\|_{\infty}+1\right)
$$

which together with (3.4) implies that there exists a positive constant $M_{1}$ such that $\left\|D_{0+}^{\alpha} x\right\|_{\infty} \leq M_{1},\left\|D_{0+}^{\alpha-1} x\right\|_{\infty} \leq B_{2}+M_{1},\left\|D_{0+}^{\alpha-2} x\right\|_{\infty} \leq B_{2}+B_{1}+M_{1}$, and $\|x\|_{\infty} \leq \frac{B_{1}+B_{2}+M_{1}}{\Gamma(\alpha-1)}$. Thus, $\|x\|_{X} \leq M$, where $M=\max \left\{B_{2}+M_{1}, B_{2}+B_{1}+M_{1}, \frac{B_{1}+B_{2}+M_{1}}{\Gamma(\alpha-1)}\right\}$.

If $x \in \Omega_{2}$, then $x(t)=c_{1} t^{\alpha-1}+c_{2} t^{\alpha-2}, c_{1}, c_{2} \in \mathbb{R}$ and $N x \in \operatorname{Im} L$. Therefore, one has $Q N\left(c_{1} t^{\alpha-1}+c_{2} t^{\alpha-2}\right)=0$ and

$$
D_{0+}^{\alpha-1} x(t)=c_{1} \Gamma(\alpha), \quad D_{0+}^{\alpha-2} x(t)=c_{1} \Gamma(\alpha) t+c_{2} \Gamma(\alpha-1)
$$

which together with $(H 2)$ and $(H 3)$ yields $\left|c_{1}\right| \leq \frac{B_{2}}{\Gamma(\alpha)}$ and $\left|c_{2}\right| \leq \frac{B_{1}+B_{2}}{\Gamma(\alpha-1)}$. Thus, $\Omega_{2}$ is bounded. 
If $x \in \Omega_{3}$, we can obtain that $x(t)=c_{1} t^{\alpha-1}+c_{2} t^{\alpha-2}, c_{1}, c_{2} \in \mathbb{R}$ and

$$
\begin{aligned}
& \lambda\left(\frac{1}{\Delta}\left(\Delta_{4} c_{1}-\Delta_{2} c_{2}\right) t^{\alpha-1}+\frac{1}{\Delta}\left(-\Delta_{3} c_{1}+\Delta_{1} c_{2}\right) t^{\alpha-2}\right) \\
& \quad+(1-\lambda)\left(\Upsilon_{1} N\left(c_{1} t^{\alpha-1}+c_{2} t^{\alpha-2}\right) t^{\alpha-1}+\Upsilon_{2} N\left(c_{1} t^{\alpha-1}+c_{2} t^{\alpha-2}\right) t^{\alpha-2}\right)=0
\end{aligned}
$$

which implies

$$
\begin{aligned}
& \lambda\left(\Delta_{4} c_{1}-\Delta_{2} c_{2}\right)+(1-\lambda)\left(\Delta_{4} \Phi_{1} N\left(c_{1} t^{\alpha-1}+c_{2} t^{\alpha-2}\right)-\Delta_{2} \Phi_{2} N\left(c_{1} t^{\alpha-1}+c_{2} t^{\alpha-2}\right)\right)=0 \\
& \lambda\left(-\Delta_{3} c_{1}+\Delta_{1} c_{2}\right)+(1-\lambda)\left(-\Delta_{3} \Phi_{1} N\left(c_{1} t^{\alpha-1}+c_{2} t^{\alpha-2}\right)+\Delta_{1} \Phi_{2} N\left(c_{1} t^{\alpha-1}+c_{2} t^{\alpha-2}\right)\right)=0 .
\end{aligned}
$$

Based on $\Delta \neq 0$, we have

$$
\begin{aligned}
& \lambda c_{1}+(1-\lambda) \Phi_{1} N\left(c_{1} t^{\alpha-1}+c_{2} t^{\alpha-2}\right)=0 \\
& \lambda c_{2}+(1-\lambda) \Phi_{2} N\left(c_{1} t^{\alpha-1}+c_{2} t^{\alpha-2}\right)=0 .
\end{aligned}
$$

For $\lambda=1$, one has $c_{1}=c_{2}=0$, which means $\Omega_{3}$ is bounded. For $\lambda=0$, in view of the first inequality of $(H 2)$ and $(H 3)$, it follows that $\Omega_{3}$ is bounded. For $\lambda \in(0,1)$, one has

$$
\begin{aligned}
& \operatorname{sgn}\left\{c_{1} \Gamma(\alpha)\right\} \lambda c_{1}+\operatorname{sgn}\left\{c_{1} \Gamma(\alpha)\right\}(1-\lambda) \Phi_{1} N\left(c_{1} t^{\alpha-1}+c_{2} t^{\alpha-2}\right)=0, \\
& \operatorname{sgn}\left\{c_{1} \Gamma(\alpha) t+c_{2} \Gamma(\alpha-1)\right\} \lambda c_{2} \\
& \quad+\operatorname{sgn}\left\{c_{1} \Gamma(\alpha) t+c_{2} \Gamma(\alpha-1)\right\}(1-\lambda) \Phi_{2} N\left(c_{1} t^{\alpha-1}+c_{2} t^{\alpha-2}\right)=0 .
\end{aligned}
$$

From (3.7) and the first inequality of (H3), it follows that $\left|c_{1}\right| \leq \frac{B_{2}}{\Gamma(\alpha)}$. Since (3.8) holds for all $t \in\left[0, \xi_{1}\right]$, by choosing $t=0$, we can obtain that

$$
\operatorname{sgn}\left\{c_{2} \Gamma(\alpha-1)\right\} \lambda c_{2}+\operatorname{sgn}\left\{c_{2} \Gamma(\alpha-1)\right\}(1-\lambda) \Phi_{2} N\left(c_{1} t^{\alpha-1}+c_{2} t^{\alpha-2}\right)=0,
$$

which together with the first inequality of $(H 2)$ yields $\left|c_{2}\right| \leq \frac{B_{1}+B_{2}}{\Gamma(\alpha-1)}$. Hence, $\Omega_{3}$ is bounded. Similarly, based on the second inequality of $(H 2)$ and $(H 3)$, we can get that

$$
\Omega_{3}^{\prime}=\left\{x \in \operatorname{Ker} L \mid-\lambda J^{-1} x+(1-\lambda) Q N x=0, \lambda \in[0,1]\right\}
$$

is bounded.

Set $\Omega=\left\{x \in X \mid\|x\|_{X}<\max \left\{M, B_{1}+2 B_{2}, \frac{B_{2}}{\Gamma(\alpha)}+\frac{B_{1}+B_{2}}{\Gamma(\alpha-1)}\right\}+1\right\}$. By the continuity of $f$, it is clear that $Q N: \bar{\Omega} \rightarrow Y$ is bounded and $K_{P}(I-Q) N: \bar{\Omega} \rightarrow X$ is compact, i.e., $N$ is $L$-compact on $\bar{\Omega}$. Moreover, from Lemma 3.1, $L$ is a Fredholm operator of index zero. Furthermore, based on the definition of $\Omega$, one has:

(i) $L x \neq \lambda N x$ for every $(x, \lambda) \in[(\operatorname{dom} L \backslash \operatorname{Ker} L) \cap \partial \Omega] \times(0,1)$;

(ii) $N x \notin \operatorname{Im} L$ for every $x \in \operatorname{Ker} L \cap \partial \Omega$.

Define

$$
H(x, \lambda)= \pm \lambda J^{-1}(x)+(1-\lambda) Q N x .
$$


Clearly, $H(x, \lambda) \neq 0$ for every $x \in \operatorname{Ker} L \cap \partial \Omega$. Hence, in view of the homotopic property of degree, it follows that

$$
\begin{aligned}
\operatorname{deg}(Q N, \Omega \cap \operatorname{Ker} L, 0) & =\operatorname{deg}(H(\cdot, 0), \Omega \cap \operatorname{Ker} L, 0)=\operatorname{deg}(H(\cdot, 1), \Omega \cap \operatorname{Ker} L, 0) \\
& =\operatorname{deg}( \pm I, \Omega \cap \operatorname{Ker} L, 0) \neq 0 .
\end{aligned}
$$

Therefore, from Lemma 2.2, we can get that $L x=N x$ admits at least one solution in $\operatorname{dom} L \cap$ $\bar{\Omega}$. Then problem (1.1) possesses at least one solution.

Corollary 3.4 Let $p(t)=p, \Delta \neq 0,(H 2),(H 3)$, and the following condition hold.

$(H 1)^{\prime}$ There exist nonnegative functions $h_{i} \in C[0,1], i=1,2,3,4$, such that, for any $t \in$ $[0,1],(x, y, z) \in \mathbb{R}^{3}$,

$$
|f(t, x, y, z)| \leq h_{1}(t)+h_{2}(t)|x|^{p-1}+h_{3}(t)|y|^{p-1}+h_{4}(t)|z|^{p-1} .
$$

Then problem (1.1) admits at least one solution, provided that

$$
\frac{2^{p}}{\Gamma(\beta+1)}\left(\frac{\left\|h_{2}\right\|_{\infty}}{(\Gamma(\alpha-1))^{p-1}}+\left\|h_{3}\right\|_{\infty}+\left\|h_{4}\right\|_{\infty}\right)<1
$$

Corollary 3.5 Let $\gamma_{1}=\gamma_{2}, \delta_{1}=\delta_{2}, \eta_{1}=\eta_{2}=1$, and $0<\xi_{1}<\xi_{2} \leq \frac{\alpha+\beta-1}{1+\alpha}<1, v_{1}>0, \alpha b_{1}+$ $a_{1}>0$. Assume that (H1), (H2), and (H3) hold. Then problem (1.1) has at least one solution, provided that (3.4) holds.

Proof From Theorem 3.3, we just need to prove $\Delta \neq 0$. In fact,

$$
\Delta=\left|\begin{array}{cc}
\Delta_{1} & \Delta_{2} \\
\Delta_{3} & \Delta_{4}
\end{array}\right|=\Delta_{1} \Delta_{4}-\Delta_{2} \Delta_{3}=\left(A_{1}-A_{2}\right)-\left(A_{3}-A_{4}\right),
$$

where

$$
\begin{aligned}
& A_{1}=\left(\frac{b_{1}}{\alpha}+\frac{a_{1}}{\alpha(1+\alpha)}\right) \frac{\xi_{2}^{\alpha+\beta-2} \Gamma(\alpha-1) \Gamma\left(\gamma_{1}+\alpha+\beta-1\right)}{\Gamma(\alpha+\beta-1) \Gamma\left(\gamma_{1}+\delta_{1}+\alpha+\beta-1\right)}, \\
& A_{2}=\frac{\nu_{1} \xi_{1}^{\alpha+\beta-1} \Gamma(\alpha) \Gamma\left(\gamma_{1}+\alpha+\beta\right)}{\Gamma(\alpha+\beta) \Gamma\left(\gamma_{1}+\delta_{1}+\alpha+\beta\right)} \cdot \frac{\xi_{2}^{\alpha+\beta-2} \Gamma(\alpha-1) \Gamma\left(\gamma_{1}+\alpha+\beta-1\right)}{\Gamma(\alpha+\beta-1) \Gamma\left(\gamma_{1}+\delta_{1}+\alpha+\beta-1\right)}, \\
& A_{3}=\left(\frac{b_{1}}{\alpha-1}+\frac{a_{1}}{\alpha(\alpha-1)}\right) \frac{\xi_{2}^{\alpha+\beta-1} \Gamma(\alpha) \Gamma\left(\gamma_{1}+\alpha+\beta\right)}{\Gamma(\alpha+\beta) \Gamma\left(\gamma_{1}+\delta_{1}+\alpha+\beta\right)}, \\
& A_{4}=\frac{\nu_{1} \xi_{1}^{\alpha+\beta-2} \Gamma(\alpha-1) \Gamma\left(\gamma_{1}+\alpha+\beta-1\right)}{\Gamma(\alpha+\beta-1) \Gamma\left(\gamma_{1}+\delta_{1}+\alpha+\beta-1\right)} \cdot \frac{\xi_{2}^{\alpha+\beta-1} \Gamma(\alpha) \Gamma\left(\gamma_{1}+\alpha+\beta\right)}{\Gamma(\alpha+\beta) \Gamma\left(\gamma_{1}+\delta_{1}+\alpha+\beta\right)} .
\end{aligned}
$$


Since $0<\xi_{1}<\xi_{2}<1$ and $\nu_{1}>0$, we have $A_{4}>A_{2}$. Next, we will prove $A_{1}>A_{3}$. Based on $\Gamma(x+1)=x \Gamma(x)$ for $x>0$ and $\delta_{1}>0$, it follows

$$
\begin{aligned}
A_{3}= & \left(\frac{b_{1}}{\alpha-1}+\frac{a_{1}}{\alpha(\alpha-1)}\right) \frac{\xi_{2} \xi_{2}^{\alpha+\beta-2}(\alpha-1)\left(\gamma_{1}+\alpha+\beta-1\right)}{(\alpha+\beta-1)\left(\gamma_{1}+\delta_{1}+\alpha+\beta-1\right)} \\
& \cdot \frac{\Gamma(\alpha-1) \Gamma\left(\gamma_{1}+\alpha+\beta-1\right)}{\Gamma(\alpha+\beta-1) \Gamma\left(\gamma_{1}+\delta_{1}+\alpha+\beta-1\right)} \\
< & \left(\frac{b_{1}}{\alpha+\beta-1}+\frac{a_{1}}{\alpha(\alpha+\beta-1)}\right) \frac{\xi_{2} \xi_{2}^{\alpha+\beta-2} \Gamma(\alpha-1) \Gamma\left(\gamma_{1}+\alpha+\beta-1\right)}{\Gamma(\alpha+\beta-1) \Gamma\left(\gamma_{1}+\delta_{1}+\alpha+\beta-1\right)},
\end{aligned}
$$

which together with $2<\alpha \leq 3,0<\beta \leq 1$, and $0<\xi_{1}<\xi_{2} \leq \frac{\alpha+\beta-1}{1+\alpha}<1$ yields that

$$
\begin{aligned}
A_{3} & <\left(\frac{b_{1}}{\alpha+\beta-1}+\frac{a_{1}}{\alpha(\alpha+\beta-1)}\right) \frac{\xi_{2} \xi_{2}^{\alpha+\beta-2} \Gamma(\alpha-1) \Gamma\left(\gamma_{1}+\alpha+\beta-1\right)}{\Gamma(\alpha+\beta-1) \Gamma\left(\gamma_{1}+\delta_{1}+\alpha+\beta-1\right)} \\
& \leq\left(\frac{b_{1}}{\alpha+\beta-1}+\frac{a_{1}}{\alpha(\alpha+\beta-1)}\right) \frac{\alpha+\beta-1}{1+\alpha} \cdot \frac{\xi_{2}^{\alpha+\beta-2} \Gamma(\alpha-1) \Gamma\left(\gamma_{1}+\alpha+\beta-1\right)}{\Gamma(\alpha+\beta-1) \Gamma\left(\gamma_{1}+\delta_{1}+\alpha+\beta-1\right)} \\
& =\left(\frac{b_{1}}{1+\alpha}+\frac{a_{1}}{\alpha(1+\alpha)}\right) \frac{\xi_{2}^{\alpha+\beta-2} \Gamma(\alpha-1) \Gamma\left(\gamma_{1}+\alpha+\beta-1\right)}{\Gamma(\alpha+\beta-1) \Gamma\left(\gamma_{1}+\delta_{1}+\alpha+\beta-1\right)}=A_{1} .
\end{aligned}
$$

Thus, we have $\Delta>0$. The proof is complete.

Example 3.6 Consider the following example:

$$
\left\{\begin{array}{l}
D_{0+}^{\frac{1}{2}} \phi_{3+\sin ^{2} t}\left(D_{0+}^{\frac{5}{2}} x(t)\right)=f\left(t, x(t), D_{0+}^{\frac{1}{2}} x(t), D_{0+}^{\frac{5}{2}} x(t)\right), \quad t \in(0,1), \\
x(0)=0, \quad \frac{2}{3} D_{0+}^{\frac{1}{2}} x(1)-\frac{4}{9} D_{0+}^{\frac{1}{2}} x(1)=\left(\frac{2}{3}\right)^{\frac{1}{2}} I_{0+, 1}^{0, \frac{1}{2}} x\left(\frac{2}{3}\right), \\
D_{0+}^{\frac{5}{2}} x(0)=0, \quad \frac{3}{4 \Gamma\left(\frac{1}{2}\right)} D_{0+}^{\alpha-2} x(0)+\frac{1}{4 \Gamma\left(\frac{1}{2}\right)} D_{0+}^{\alpha-1} x(0)=\left(\frac{3}{4}\right)^{\frac{1}{2}} I_{0+, 1}^{\frac{1}{2}, 1} x\left(\frac{3}{4}\right),
\end{array}\right.
$$

where $\beta=\frac{1}{2}, \alpha=\frac{5}{2}, p(t)=3, \gamma_{1}=0, \gamma_{2}=\frac{1}{2}, \eta_{1}=\eta_{2}=1, \delta_{1}=\frac{1}{2}, \delta_{2}=1, \xi_{1}=\frac{2}{3}, \xi_{2}=\frac{3}{4}, v_{1}=$ $\left(\frac{2}{3}\right)^{\frac{1}{2}}, v_{2}=\left(\frac{3}{4}\right)^{\frac{1}{2}}, a_{1}=\frac{2}{3}, a_{2}=\frac{3}{4 \Gamma\left(\frac{1}{2}\right)}, b_{1}=-\frac{4}{9}, b_{2}=\frac{1}{4 \Gamma\left(\frac{1}{2}\right)}$,

$$
f(t, x, y, z)= \begin{cases}\frac{t^{2}}{32}+\frac{t^{2}}{32} \sin ^{2} x+\frac{\left(t-\frac{2}{3}\right)^{2}}{32} \sin ^{2} z+1, & t \in\left[0, \frac{2}{3}\right], x, z \in \mathbb{R} \\ \frac{t^{2}}{32}+\frac{t^{2}}{32} \sin ^{2} x+1, & t \in\left(\frac{2}{3}, \frac{3}{4}\right], x \in \mathbb{R} \\ \frac{t^{2}}{32}+\frac{t^{2}}{32} \sin ^{2} x+\frac{\left(t-\frac{3}{4}\right)^{2}}{8} y^{2}+1, & t \in\left(\frac{3}{4}, 1\right], x, y \in \mathbb{R} .\end{cases}
$$

By simple calculation, it is clear that $\Delta \neq 0$ and $(H)$ is satisfied. Moreover,

$$
|f(t, x, y, z)| \leq h_{1}(t)+h_{2}(t)|x|^{2}+h_{3}(t)|y|^{2}+h_{4}(t)|z|^{2},
$$

where $h_{1}(t)=\frac{t^{2}}{32}+1, h_{2}(t)=\frac{t^{2}}{32}$,

$$
h_{3}(t)=\left\{\begin{array}{ll}
0, & t \in\left[0, \frac{3}{4}\right], \\
\frac{\left(t-\frac{3}{4}\right)^{2}}{8}, & t \in\left(\frac{3}{4}, 1\right] ;
\end{array} \quad h_{4}(t)= \begin{cases}\frac{\left(t-\frac{2}{3}\right)^{2}}{32}, & t \in\left[0, \frac{2}{3}\right], \\
0, & t \in\left(\frac{2}{3}, 1\right]\end{cases}\right.
$$

which implies that (3.4) and (H1) hold. Let $D_{0_{+}}^{\frac{1}{2}} x(t)>1$ for any $t \in\left[\frac{3}{4}, 1\right]$. Since $f(t, x, y, z)>$ 0 , we have $\operatorname{sgn}\left\{D_{0_{+}}^{\frac{1}{2}} x(t)\right\} \Phi_{2} N x(t)>0$. Similarly, if $D_{0+}^{\frac{1}{2}} x(t)<-1$ for any $t \in\left[\frac{3}{4}, 1\right]$, it follows 
$\operatorname{sgn}\left\{D_{0+}^{\frac{1}{2}} x(t)\right\} \Phi_{2} N x(t)<0$. Hence, $(H 2)$ is satisfied. If $D_{0+}^{\frac{3}{2}} x(t)>1$ for any $t \in\left[0, \frac{2}{3}\right]$, we have $1<f(t, x, y, z)<\frac{75}{72}$ and

$$
\left(\frac{2 t^{\frac{1}{2}}}{\Gamma\left(\frac{1}{2}\right)}\right)^{\frac{1}{2}}<N x(t)<\left(\frac{150 t^{\frac{1}{2}}}{72 \Gamma\left(\frac{1}{2}\right)}\right)^{\frac{1}{2}} .
$$

Since

$$
\begin{aligned}
- & \frac{4}{9} \int_{0}^{1} N x(s) d s+\frac{2}{3} \int_{0}^{1}(1-s) N x(s) d s \\
& <-\frac{4}{9}\left(\frac{2}{\Gamma\left(\frac{1}{2}\right)}\right)^{\frac{1}{2}} \int_{0}^{1} s^{\frac{1}{4}} d s+\frac{2}{3}\left(\frac{150}{72 \Gamma\left(\frac{1}{2}\right)}\right)^{\frac{1}{2}} \int_{0}^{1}(1-s) s^{\frac{1}{4}} d s \\
& =-\frac{16}{45}\left(\frac{2}{\Gamma\left(\frac{1}{2}\right)}\right)^{\frac{1}{2}}+\frac{32}{135}\left(\frac{150}{72 \Gamma\left(\frac{1}{2}\right)}\right)^{\frac{1}{2}}<0
\end{aligned}
$$

and $-\left(\frac{2}{3}\right)^{\frac{1}{2}} I_{0+, 1}^{0, \frac{1}{2}} I_{0+}^{\frac{5}{2}} N x\left(\frac{2}{3}\right)<0$, we can obtain $\operatorname{sgn}\left\{D_{0+}^{\frac{3}{2}} x(t)\right\} \Phi_{1} N x(t)<0$. Similarly, if $D_{0_{+}}^{\frac{3}{2}} x(t)<$ -1 for any $t \in\left[0, \frac{2}{3}\right]$, one has $\operatorname{sgn}\left\{D_{0+}^{\frac{1}{2}} x(t)\right\} \Phi_{1} N x(t)>0$. Therefore, $(H 3)$ is verified. Based on the above facts, problem (3.10) has at least one solution.

\section{Conclusions}

This paper is concerned with the solvability for Erdélyi-Kober fractional integral boundary value problems with $p(t)$-Laplacian operator at resonance. By employing the coincidence degree method of Mawhin, some new results on the existence of solutions are acquired. It should be emphasized that the Erdélyi-Kober fractional integral operator is a generalization of the integral of integer order and can convert into Riemann-Liouville fractional integral with a power weight when $\eta=1$ and $\gamma=0$. So, a problem of this type becomes more interesting and challenging. Moreover, we consider the Erdélyi-Kober fractional integral boundary value problems with $p(t)$-Laplacian operator at resonance when the dimension of the kernel of operator $D_{0^{+}}^{\beta} \varphi_{p(t)}\left(D_{0^{+}}^{\alpha} x\right)$ is equal to 2 , which causes a lot of difficulties such as constructing continuous linear projections. Thus, our results extend and enrich some existing papers. Furthermore, there are few papers studying fractional integral boundary value problem with $p(t)$-Laplacian operator.

Acknowledgements

The authors wish to express their sincere appreciation to the editor and referees for their useful suggestions which have significantly improved the paper.

\section{Funding}

This work is supported by the Research Initiation Foundation of Xuzhou Medical University (N0. D2019003) and the Natural Science Foundation of Jiangsu Province (No. BK20190620).

\section{Availability of data and materials}

Data sharing not applicable to this article as no data sets were generated or analysed during the current study.

\section{Competing interests}

The authors declare that they have no competing interests.

\section{Authors' contributions}

The authors contributed equally in this article. They have all read and approved the final manuscript.

\section{Author details}

${ }^{1}$ School of Public Health, Xuzhou Medical University, Xuzhou 221004, P.R. China. ${ }^{2}$ School of Mathematics, China University of Mining and Technology, Xuzhou 221116, P.R. China. 


\section{Publisher's Note}

Springer Nature remains neutral with regard to jurisdictional claims in published maps and institutional affiliations.

\section{Received: 5 June 2020 Accepted: 29 September 2020 Published online: 08 October 2020}

\section{References}

1. Killbas, A.A., Srivastava, H.M., Trujillo, J..: Theory and Applications of Fractional Differential Equations. North-Holland Mathematics Studies, vol. 204. Elsevier, Amsterdam (2006)

2. Leszczynski, J., Blaszczyk, T.: Modeling the transition between stable and unstable operation while emptying a silo. Granul. Matter 13, 429-438 (2011)

3. Bai, J., Feng, X.: Fractional-order anisotropic diffusion for image denoising. IEEE Trans. Image Process. 16, 2492-2502 (2007)

4. Podlubny, I.: Fractional Differential Equations. Academic Press, New York (1999)

5. Magin, R.: Fractional calculus models of complex dynamics in biological tissues. Comput. Math. Appl. 59, 1586-1593 (2010)

6. Chen, Y., Levine, S., Rao, M.: Variable exponent, linear growth functionals in image restoration. SIAM J. Appl. Math. 66 , 1383-1406 (2006)

7. Szymanek, E.: The application of fractional order differential calculus for the description of temperature profiles in a granular layer. In: Mitkowski, W., Kacprzyk, J., Baranowski, J. (eds.) Advances in the Theory and Applications of Non-integer Order Systems. LNEE, vol. 275. Springer, Cham (2013)

8. Ahmad, B., Nieto, J.J.: Existence of solutions for anti-periodic boundary value problems involving fractional differential equations via Leray-Schauder degree theory. Topol. Methods Nonlinear Anal. 35(2), 295-304 (2010)

9. Ahmad, B., Ntouyas, S.K.: On perturbed fractional differential inclusions with nonlocal multi-point Erdélyi-Kober fractional integral boundary conditions. Mediterr. J. Math. 14(1), Article ID 27 (2017)

10. Cabada, A., Wang, G.: Positive solutions of nonlinear fractional differential equations with integral boundary value conditions. J. Math. Anal. Appl. 389, 403-411 (2012)

11. Hu, Z., Liu, W.: Solvability for fractional order boundary value problem at resonance. Bound. Value Probl. 2011, Article ID 20 (2011)

12. Bai, Z:: Solvability for a class of fractional m-point boundary value problem at resonance. Comput. Math. Appl. 62 1292-1302 (2011)

13. Kosmatov, N.: A boundary value problem of fractional order at resonance. Electron. J. Differ. Equ. 2010, Article ID 135 (2010)

14. Jiang, W.: The existence of solutions to boundary value problems of fractional differential equations at resonance. Nonlinear Anal. 74, 1987-1994 (2011)

15. Nyamoradi, N., Tayyebi, E.: Existence of solutions for a class of fractional boundary value equations with impulsive effects via critical point theory. Mediterr. J. Math. 15, Article ID 79 (2018)

16. Jiao, F., Zhou, Y.: Existence results for fractional boundary value problem via critical point theory. Int. J. Bifurc. Chaos 22(4), Article ID 1250086 (2012)

17. Ge, W., Ren, J.: An extension of Mawhin's continuation theorem and its application to boundary value problems with a p-Laplacian. Nonlinear Anal. 58, 477-488 (2004)

18. Lu, S., Gui, Z:: On the existence of periodic solutions to $p$-Laplacian Rayleigh differential equation with a delay. J. Math. Anal. Appl. 325(1), 685-702 (2007)

19. Pang, H., Ge, W., Tian, M.: Solvability of nonlocal boundary value problems for ordinary differential equation of higher order with a $p$-Laplacian. Comput. Math. Appl. 56, 127-142 (2008)

20. Mahmudov, N.I., Unul, S.: Existence of solutions of fractional boundary value problems with $p$-Laplacian operator. Bound. Value Probl. 2015, Article ID 99 (2015)

21. Chen, T., Liu, W., Hu, Z:: A boundary value problem for fractional differential equation with $p$-Laplacian operator at resonance. Nonlinear Anal. 75, 3210-3217 (2012)

22. Chen, T., Liu, W.: An anti-periodic boundary value problem for the fractional differential equation with a $p$-Laplacian operator. Appl. Math. Lett. 25, 1671-1675 (2012)

23. Chai, G.: Positive solutions for boundary value problem of fractional differential equation with $p$-Laplacian operator. Bound. Value Probl. 2012, Article ID 18 (2012)

24. Shen, T., Liu, W.: Existence of solutions for fractional integral boundary value problems with $p(t)$-Laplacian operator. J. Nonlinear Sci. Appl. 9, 5000-5010 (2016)

25. Pélissier, M.C., Reynaud, M.L.: Etude d'un modèle mathématique d'écoulement de glacier. C. R. Acad. Sci. Paris, Ser. I 279, 531-534 (1974)

26. Kawohl, B.: On a family of torsional creep problems. J. Reine Angew. Math. 410, 1-22 (1990)

27. Leibenson, L.: General problem of the movement of a compressible fluid in a porous medium. Izv. Akad. Nauk Kirg. SSR Geogr. Geophys. 9, 7-10 (1983)

28. Fan, X., Zhang, Q., Zhao, D.: Eigenvalues of $p(x)$-Laplacian Dirichlet problem. J. Math. Anal. Appl. 302, 306-317 (2005)

29. Zhang, Q., Wang, Y., Qiu, Z.: Existence of solutions and boundary asymptotic behavior of $p(r)$-Laplacian equation multi-point boundary value problems. Nonlinear Anal. 72, 2950-2973 (2010)

30. Mawhin, J.: Topological degree and boundary value problems for nonlinear differential equations in topological methods for ordinary differential equations. In: Topological Methods for Ordinary Differential Equations. Lecture Notes in Math., vol. 1537, pp. 74-142. Springer, Berlin (1993)

31. Kober, H.: On fractional integrals and derivatives. Q. J. Math. Oxf. Ser. (2) 1, 193-211 (1940) 